\title{
Constructing Canonical Bases of Quantized Enveloping Algebras
}

\author{
Willem A. de Graaf
}

\section{CONTENTS}

1. Introduction

2. The Canonical Basis

3. Kashiwara Operators

4. Adapted Strings

5. Constructing Canonical Basis Elements

6. Canonical Bases of Modules

7. Tight Monomials of Small Weight

References

2000 AMS Subject Classification: Primary 17B37; Secondary 68W30

Keywords: quantum groups, canonical bases, algorithms
An algorithm for computing the elements of a given weight of the canonical basis of a quantized enveloping algebra is described. Subsequently, a similar algorithm is presented for computing the canonical basis of a finite-dimensional module.

\section{INTRODUCTION}

Since the invention of canonical bases of quantized enveloping algebras, one of the main problems has been to establish what they look like. Explicit formulas are only known in a few cases corresponding to root systems of low rank, namely $A_{1}$ (trivial), $A_{2}$ ([Lusztig 90]), $A_{3}$ ([Xi 99a]), and $B_{2}$ ([Xi 99b]). Furthermore, there is evidence suggesting that for higher ranks the formulas become so complicated that an explicit description is virtually impossible (see [Carter 97]). Therefore, it is natural to start less ambitiously, and try to find part of the canonical basis, say the part consisting of all elements of a given weight. We will describe an algorithm for computing the elements of a given weight of the canonical basis of the negative part of the quantized enveloping algebra of a finite-dimensional semisimple Lie algebra. We will also give an algorithm for computing the canonical basis of a finite-dimensional module.

To the best of my knowledge, two algorithms for computing canonical bases of modules are known. In [Leclerc and Toffin 00] an algorithm is described for computing the canonical basis of a $U_{q}\left(\mathfrak{s l}_{n}\right)$-module, and [Marsh 96] contains an algorithm for computing the canonical basis of a fundamental module, when the root system is of type $A-D$. Our approach differs from the ones taken in [Leclerc and Toffin 00], [Marsh 96] in that we work with PBW-type bases. This leads to algorithms that are more generally applicable: They work for any finitedimensional module of the quantized enveloping algebra

(c) A K Peters, Ltd $1058-6458 / 2001 \$ 0.50$ per page Experimental Mathematics 11:2, page 161 
of any semisimple Lie algebra. The problem of computing elements of the canonical basis of the negative part of a quantized enveloping algebra has, to the best of my knowledge, not been considered before.

This paper is organized as follows. In Section 2 we recall some basic facts on canonical bases. In Section 3 we describe an algorithm for computing the action of the Kashiwara operators. In Section 4 we describe the notion of adapted string, following [Littleman 98]. Then, in Section 5, we describe an algorithm for computing the elements of the canonical basis of a given weight $\nu$. In Section 6 we give a similar algorithm for computing the canonical basis of a finite-dimensional $U_{q}(\mathfrak{g})$-module. Finally, in Section 7, we apply the algorithm of Section 5 to the study of tight monomials of small weight.

The algorithms described here have been implemented in the computer algebra system GAP4 ([GAP 00]), as part of a package called QuaGroup, [Graaf 01b]. The main computations for Examples 5.2, 5.3 and for Table 1 were done using that package.

\section{THE CANONICAL BASIS}

First we recall some notation. Our main reference is [Jantzen 96a]. Let $\mathfrak{g}$ be a finite-dimensional semisimple Lie algebra with root system $\Phi$. Let $\Delta=\left\{\alpha_{1}, \ldots, \alpha_{l}\right\}$ be a simple system of $\Phi$. Then $U_{q}(\mathfrak{g})$ is the corresponding quantized enveloping algebra, with generators $F_{\alpha}, K_{\alpha}^{ \pm 1}$, $E_{\alpha}$, for $\alpha \in \Delta$, subject to the relations [Jantzen 96a, 4.3]. Furthermore, $U^{-}$will be the subalgebra of $U_{q}(\mathfrak{g})$ generated by the $F_{\alpha}$. Let $\nu=\sum_{i=1}^{l} k_{i} \alpha_{i}$ be a linear combination of the simple roots, with non-negative integral coefficients. Then $U_{-\nu}^{-}$will be the span of all elements $F_{\alpha_{m_{1}}} \cdots F_{\alpha_{m_{t}}}$ such that $F_{\alpha_{i}}$ appears exactly $k_{i}$ times. Elements of $U_{-\nu}^{-}$are said to be homogeneous of weight $\nu$.

By $W(\Phi)$, we denote the Weyl group of $\Phi$. It is generated by the simple reflections $s_{i}=s_{\alpha_{i}}$ for $1 \leq i \leq l$. Usually, we will denote a reduced expression for the longest element in $W(\Phi)$ by $w_{0}$.

We work in the subalgebra $U^{-}$of $U_{q}(\mathfrak{g})$. Let $w_{0}=$ $s_{i_{1}} \cdots s_{i_{t}}$ be a reduced expression for the longest element in the Weyl group. For $1 \leq k \leq l$, let $T_{k}=T_{\alpha_{k}}: U_{q}(\mathfrak{g}) \rightarrow$ $U_{q}(\mathfrak{g})$ be the automorphism described in [Jantzen 96a, 8.13]. For $1 \leq k \leq t$, set $F_{k}=T_{i_{1}} \cdots T_{i_{k-1}}\left(F_{\alpha_{i_{k}}}\right)$. Then $F_{k}$ is an element of weight $\beta_{k}=s_{i_{1}} \cdots s_{i_{k-1}}\left(\alpha_{i_{k}}\right)$. We also denote $F_{k}$ by $F_{\beta_{k}}$. As usual, we set $F_{k}^{(m)}=$ $F_{k}^{m} /[m] !_{\alpha_{i_{k}}}$ (where $[m] !_{\alpha_{i_{k}}}$ is the Gaussian factorial, defined in [Jantzen 96a, 0.1, 4,2]). Then the monomials

$$
F_{1}^{\left(n_{1}\right)} \cdots F_{t}^{\left(n_{t}\right)}
$$

form a basis of $U^{-}$. This basis is called a PBW-type basis; we call a monomial of the form $(2-1)$ a PBWmonomial (relative to the chosen reduced expression for the longest element of the Weyl group). We have algorithms for writing the product of any two PBWmonomials as a linear combination of PBW-monomials ([Graaf 01a]).

Let $x$ be a monomial of the form $(2-1)$. To stress the dependency of $x$ on the choice of reduced expression for the longest element of the Weyl group, we say that $x$ is a $w_{0}$-monomial. We refer to the exponents $n_{1}, \ldots, n_{t}$ as the first, second, $\ldots, t^{\text {th }}$ exponent of $x$.

Now we let ${ }^{-}$be the unique automorphism of $U^{-}$ (viewed as a $\mathbb{Q}$-algebra) satisfying $\bar{q}=q^{-1}$ and $\bar{F}_{\alpha_{i}}=$ $F_{\alpha_{i}}$. Elements that are invariant under ${ }^{-}$are said to be bar-invariant. The bar-invariant elements include all monomials of the form $F_{\alpha_{i_{1}}}^{\left(n_{1}\right)} \cdots F_{\alpha_{i_{r}}}^{\left(n_{r}\right)}$ (but not all PBWmonomials).

By results of Lusztig ([Lusztig 93a, Theorem 42.1.10], [Lusztig 96, Proposition 8.2], see also [Berenstein and Zelevinsky 01]), there is a unique basis $\mathbf{B}$ of $U^{-}$with the following properties:

Firstly, all elements of B are bar-invariant. Secondly, for any choice of reduced expression $w_{0}$ for the longest element in the Weyl group, and any element $X \in \mathbf{B}$, $X=x+\sum \zeta_{i} x_{i}$, where $x, x_{i}$ are different $w_{0}$-monomials, and $\zeta_{i} \in q \mathbb{Z}[q]$.

The basis $\mathbf{B}$ is called the canonical basis. If we work with a reduced expression $w_{0}$ for the longest element in $W(\Phi)$, and write $X \in \mathbf{B}$ as above, then we say that $x$ is the principal $w_{0}$-monomial of $X$ (or just principal monomial of $X$, if it is clear which reduced expression we mean).

We let $\mathcal{L}(\infty)$ be the $\mathbb{Z}[q]$-lattice spanned by $\mathbf{B}$. Fix a reduced expression $w_{0}$ for the longest element in $W(\Phi)$. Then $\mathcal{L}(\infty)$ is also spanned by the set of all $w_{0}$-monomials (cf. [Lusztig 93a, Chapter 42]; it will also follow from Proposition 5.1 in Section 5 ). We let $\pi: \mathcal{L}(\infty) \rightarrow \mathcal{L}(\infty) / q \mathcal{L}(\infty)$ be the projection map, and we let $\mathcal{B}(\infty)$ be the set of all $\pi(x)$, where $x$ runs through all $w_{0}$-monomials. Then $\mathcal{B}(\infty)$ does not depend on the choice of reduced expression for the longest element in $W(\Phi)$. (Indeed, let $\widetilde{w}_{0}$ be a second reduced expression for the longest element in $W(\Phi)$. Let $x$ be a $w_{0}$-monomial, and let $X$ be the element of $\mathbf{B}$ with principal $w_{0}$-monomial $x$. Let $x^{\prime}$ be its principal $\widetilde{w}_{0}$-monomial, then $x=x^{\prime} \bmod q \mathcal{L}(\infty)$.)

Let $x$ be a $w_{0}$-monomial, then we write $b_{x}=\pi(x) \in$ $\mathcal{B}(\infty)$. Also $G\left(b_{x}\right)$ will denote the element of $\mathbf{B}$ which has principal monomial $x$, i.e., such that $\pi\left(G\left(b_{x}\right)\right)=b_{x}$. 
Remark 2.1. In this section, we have worked with a $\mathbb{Z}[q]$ lattice $\mathcal{L}(\infty)$ in $U^{-}$. However, in some other places (e.g., [Berenstein and Zelevinsky 01], [Lusztig 93a], [Lusztig 96]) a $\mathbb{Z}\left[q^{-1}\right]$-lattice in $U^{+}$is used. In these references, a different PBW-type basis is used (compare the description of $T_{\alpha}$ in [Jantzen 96a, 8.14] with the description of $T_{i,-1}^{\prime}$ in [Lusztig 93a, 37.1.3]). The two approaches are equivalent. In order to see that, we view $U_{q}(\mathfrak{g})$ as a $\mathbb{Q}$-algebra, and we let $\phi$ be the automorphism of $U_{q}(\mathfrak{g})$ defined by $\phi\left(F_{\alpha}\right)=E_{\alpha}, \phi\left(K_{\alpha}\right)=K_{\alpha}, \phi\left(E_{\alpha}\right)=F_{\alpha}$, and $\phi(q)=q^{-1}$. (This is the composition of the automorphisms $\omega$ of [Jantzen 96a, Lemma 4.6], and $\psi$ of [Jantzen 96a, Proposition 11.9].) Then $\phi\left(U^{+}\right)=U^{-}$and $\phi\left(T_{i,-1}^{\prime}(u)\right)=T_{\alpha}(\phi(u))$ for all $u \in U_{q}(\mathfrak{g})$. Therefore $\phi$ maps a PBW-type basis of $U^{+}$(defined using the $T_{i,-1}^{\prime}$ ) to a PBW-type basis of $U^{-}$(defined using the $T_{\alpha}$ ), and interchanges $q$ and $q^{-1}$. Also, $\phi$ maps bar-invariant elements of $U^{+}$to bar-invariant elements of $U^{-}$, so that $\phi$ maps the canonical basis of $U^{+}$to the canonical basis of $U^{-}$.

\section{KASHIWARA OPERATORS}

Let $\alpha \in \Delta$. The Kashiwara operators $\widetilde{F}_{\alpha}, \widetilde{E}_{\alpha}: U^{-} \rightarrow U^{-}$ are defined as follows. Let $w_{0}=s_{i_{1}} \cdots s_{i_{t}}$ be a reduced expression for the longest element of the Weyl group, such that $\alpha_{i_{1}}=\alpha$. Let $u$ be a $w_{0}$-monomial with exponents $n_{1}, \ldots, n_{t}$. Then $\widetilde{F}_{\alpha}(u)=F_{1}^{\left(n_{1}+1\right)} \cdots F_{t}^{\left(n_{t}\right)}$, and $\widetilde{E}_{\alpha}(u)=F_{1}^{\left(n_{1}-1\right)} \cdots F_{t}^{\left(n_{t}\right)}$, if $n_{1}>0$, and $\widetilde{E}_{\alpha}(u)=0$ otherwise. (Note that $F_{1}=F_{\alpha}$.) The action of $\widetilde{F}_{\alpha}, \widetilde{E}_{\alpha}$ is extended to the whole of $U^{-}$by linearity. It can be shown that this definition does not depend on the choice of reduced expression of the longest element in the Weyl group (cf. [Jantzen 96a, 10.1]).

Then $\widetilde{F}_{\alpha}$ and $\widetilde{E}_{\alpha}$ map PBW-monomials to PBWmonomials, relative to a reduced expression for the longest element in $W(\Phi)$ starting with $s_{\alpha}$. However, $\mathcal{B}(\infty)$ does not depend on that choice, and therefore, $\widetilde{F}_{\alpha}$ and $\widetilde{E}_{\alpha}$ can be viewed as maps $\widetilde{F}_{\alpha}: \mathcal{B}(\infty) \rightarrow \mathcal{B}(\infty)$ and $\widetilde{E}_{\alpha}: \mathcal{B}(\infty) \rightarrow \mathcal{B}(\infty) \cup\{0\}$. This means that if $x$ is a $w_{0}$-monomial, then $\widetilde{F}_{\alpha}(x)=x^{\prime} \bmod q \mathcal{L}(\infty)$, where $x^{\prime}$ is a certain $w_{0}$-monomial. We consider the problem of obtaining $x^{\prime}$ from $x$.

First we note that if $w_{0}$ happens to start with $s_{\alpha}$, then $x^{\prime}$ is constructed from $x$ by increasing the first exponent of $x$ by 1 . Now suppose that $w_{0}$ does not start with $s_{\alpha}$. Let $\widetilde{w}_{0}$ be a different reduced expression for the longest element of the Weyl group. Then there is a $\widetilde{w}_{0}$-monomial $\tilde{x}$ such that $x=\tilde{x} \bmod q \mathcal{L}(\infty)$. Following Lusztig's notation (see [Lusztig 92], [Lusztig 93a]), we write $\tilde{x}=R_{w_{0}}^{\tilde{w}_{0}}(x)$. If we can find $\tilde{x}$ from $x$, then the problem of calculating $\widetilde{F}_{\alpha}(x)$ is solved. Indeed, let $\widetilde{w}_{0}$ be a reduced expression for the longest element of the Weyl group, starting with $s_{\alpha}$. We find $\tilde{x}=R_{w_{0}}^{\tilde{w}_{0}}(x)$, and increase its first exponent by 1 . Denote the resulting monomial by $\tilde{x}^{\prime}$. Finally, we construct $x^{\prime}=R_{\tilde{w}_{0}}^{w_{0}}\left(\tilde{x}^{\prime}\right)$. Then $\widetilde{F}_{\alpha}(x)=x^{\prime} \bmod q \mathcal{L}(\infty)$.

We may assume that $\widetilde{w}_{0}$ can be obtained from $w_{0}$ by applying one braid relation. Suppose that this relation amounts to replacing $s_{\alpha} s_{\beta} \cdots$ by $s_{\beta} s_{\alpha} \cdots$, where both words are of length $d$. Then $d=2,3,4$ or 6 . Suppose that the first word occurs in $w_{0}$ on positions $p, p+1, \ldots, p+$ $d-1$. Write $x=F_{1}^{\left(m_{1}\right)} \cdots F_{t}^{\left(m_{t}\right)}$ and $\tilde{x}=F_{1}^{\left(m_{1}^{\prime}\right)} \cdots F_{t}^{\left(m_{t}^{\prime}\right)}$ (where the $F_{i}$ in $\tilde{x}$ are defined relative to $\widetilde{w}_{0}$ ). We obtain the $m_{i}^{\prime}$ from the $m_{i}$ in the following way:

1. If $d=2$, then $m_{p}^{\prime}=m_{p+1}$ and $m_{p+1}^{\prime}=m_{p}$.

2. If $d=3$, set $\mu=\min \left(m_{p}, m_{p+2}\right)$, and $m_{p}^{\prime}=m_{p+1}+$ $m_{p+2}-\mu, m_{p+1}^{\prime}=\mu, m_{p+2}^{\prime}=m_{p}+m_{p+1}-\mu$.

3. If $d=4$, suppose that the move consists of replacing $s_{\alpha} s_{\beta} s_{\alpha} s_{\beta}$ by $s_{\beta} s_{\alpha} s_{\beta} s_{\alpha}$. Set $a=m_{p}, b=m_{p+1}$, $c=m_{p+2}, d=m_{p+3}$.

(a) If $\alpha$ is short, then set $n_{1}=\max (b, \max (b, d)+$ $c-a), n_{2}=\max (a, c)+2 b, n_{3}=\min (c+$ $d, a+\min (b, d)), n_{4}=\min (a, c)$. Set $\mu=$ $\max \left(2 n_{3}, n_{2}+n_{4}\right)$ and $m_{p}^{\prime}=n_{1}, m_{p+1}^{\prime}=\mu-n_{2}$, $m_{p+2}^{\prime}=n_{2}+n_{3}-\mu, m_{p+3}^{\prime}=n_{4}-2 n_{3}+\mu$.

(b) If $\alpha$ is long, then set $p_{1}=\max (b, \max (b, d)+$ $2 c-2 a), p_{2}=\max (a, c)+b, p_{3}=\min (2 c+$ $d, \min (b, d)+2 a), p_{4}=\min (a, c)$. Set $\mu=$ $\max \left(p_{3}, p_{2}+p_{4}\right)$, and $m_{p}^{\prime}=p_{1}, m_{p+1}^{\prime}=\mu-p_{2}$, $m_{p+2}^{\prime}=p_{3}+2 p_{2}-2 \mu, m_{p+3}^{\prime}=p_{4}-p_{3}+\mu$.

4. If $d=6$, we consider the root system of type $D_{4}$, along with its diagram automorphism $\phi$ of order 3 . Let $\alpha_{2}$ be the simple root fixed by $\phi$, and $\alpha_{1}, \alpha_{3}, \alpha_{4}$ the other three. Set $v=s_{1} s_{3} s_{4}$. We use the following two reduced expressions for the longest element in the Weyl group: $v_{0}=v s_{2} v s_{2} v s_{2}$ and $\widetilde{v}_{0}=s_{2} v s_{2} v s_{2} v$. Let $\widetilde{U}^{-}$be the algebra generated by the $F_{\alpha_{i}}$ for $1 \leq i \leq 4$, of the corresponding quantized enveloping algebra. In $\widetilde{U}^{-}$, we use the PBW-bases relative to $v_{0}$ and $\widetilde{v}_{0}$.

For simplicity assume that the root system of $U_{q}(\mathfrak{g})$ is of type $G_{2}$. Suppose that the braid relation amounts to replacing $w_{0}=s_{\alpha} s_{\beta} s_{\alpha} s_{\beta} s_{\alpha} s_{\beta}$ by $\widetilde{w}_{0}=s_{\beta} s_{\alpha} s_{\beta} s_{\alpha} s_{\beta} s_{\alpha}$, where $\alpha$ is long. Corresponding to a $w_{0}$-monomial 
$x$ with exponents $m_{1}, \ldots, m_{6}$, we construct the $v_{0}$-monomial $y=\psi_{1}(x)$ with exponents $m_{1}, m_{1}, m_{1}, m_{2}, m_{3}, m_{3}, m_{3}, m_{4}, m_{5}, m_{5}, m_{5}, m_{6}$.

Furthermore, corresponding to a $\widetilde{w}_{0}$-monomial $\tilde{x}$ with exponents $m_{1}, \ldots, m_{6}$ we construct the $\widetilde{v}_{0}$-monomial $\tilde{y}=\psi_{2}(\tilde{x})$ with exponents $m_{1}, m_{2}, m_{2}, m_{2}, m_{3}, m_{4}, m_{4}, \quad m_{4}, m_{5}, m_{6}, m_{6}, m_{6}$. Now starting with a $w_{0}$-monomial $x$, we construct (using Cases 1 and 2) the $\widetilde{v}_{0}$-monomial $\tilde{y}=R_{v_{0}}^{\tilde{v}_{0}}\left(\psi_{1}(x)\right)$. Then we have $R_{w_{0}}^{\tilde{w}_{0}}(x)=\psi_{2}^{-1}(\tilde{y})$.

Finally, if $\alpha$ is short, we have $R_{w_{0}}^{\tilde{w}_{0}}(x)=$ $\psi_{1}^{-1}\left(R_{\tilde{v}_{0}}^{v_{0}}\left(\psi_{2}(x)\right)\right)$.

Cases 1 and 2 are proved in [Lusztig 93a]; Case 3 can be proved using [Lusztig 92, 12.5], and Case 4 follows in the same way (see also [Carter 97], [Lusztig 93a, Theorem 14.4.9]). At the end of Section 5, we sketch a different proof of Cases 2 and 3.

We note that by the same methods we can calculate the action of $\widetilde{E}_{\alpha}$.

Example 3.1. Consider the root system of type $A_{3}$, with simple roots $\alpha, \beta, \gamma$ (where $\alpha, \gamma$ correspond to the outer vertices of the Dynkin diagram of type $A_{3}$, and $\beta$ to the middle vertex). Then $w_{0}=s_{\alpha} s_{\beta} s_{\gamma} s_{\alpha} s_{\beta} s_{\alpha}$ is a reduced expression for the longest element in the Weyl group. Let $x$ be the $w_{0}$-monomial with exponents $(1,2,3,4,5,6)$. We calculate the action of $\widetilde{F}_{\gamma}$ on $x$. First of all, $\widetilde{w}_{0}=s_{\gamma} s_{\alpha} s_{\beta} s_{\gamma} s_{\alpha} s_{\beta}$ is a reduced expression for the longest element in the Weyl group, starting with $s_{\gamma}$. By applying braid relations, $w_{0}$ is transformed to $\widetilde{w}_{0}$ in the following way:

$$
\begin{aligned}
w_{0} \rightarrow s_{\alpha} s_{\beta} s_{\gamma} s_{\beta} s_{\alpha} s_{\beta} & \rightarrow s_{\alpha} s_{\gamma} s_{\beta} s_{\gamma} s_{\alpha} s_{\beta} \\
& \rightarrow s_{\gamma} s_{\alpha} s_{\beta} s_{\gamma} s_{\alpha} s_{\beta}=\widetilde{w}_{0}
\end{aligned}
$$

Using Cases 1 and 2, we see that $x$ transforms to a monomial with exponents $(1,2,3,7,4,5),(1,8,2,3,4,5)$, and $(8,1,2,3,4,5)$. Here the last sequence of exponents defines the $\widetilde{w}_{0}$-monomial $\tilde{x}$. We now increase its first exponent to 9 , obtaining the $\widetilde{w}_{0}$-monomial $\tilde{x}^{\prime}$. Transforming this back we obtain the $w_{0}$-monomial $x^{\prime}$ with exponents $(1,2,3,4,5,7)$, which is equal to $\widetilde{F}_{\gamma}(x)$.

\section{ADAPTED STRINGS}

First we recall some facts on Littelmann's path model. For more details and proofs, we refer to [Littleman 95].

Let $P$ denote the weight lattice, and let $P_{\mathbb{R}}$ be the vector space over $\mathbb{R}$ spanned by $P$ (i.e., $P_{\mathbb{R}}=P \otimes_{\mathbb{Z}} \mathbb{R}$ ). Let $\Pi$ be the set of all piecewise linear paths $\xi:[0,1] \rightarrow P_{\mathbb{R}}$, such that $\xi(0)=0$. For $\alpha \in \Delta$ Littelmann defines operators $f_{\alpha}, e_{\alpha}: \Pi \rightarrow \Pi \cup\{0\}$. Let $\lambda$ be a dominant weight and let $\xi_{\lambda}$ be the path joining $\lambda$ and the origin by a straight line. Let $\Pi_{\lambda}$ be the set of all nonzero $f_{\alpha_{i_{1}}} \cdots f_{\alpha_{i_{m}}}\left(\xi_{\lambda}\right)$ for $m \geq 0$. Then $\xi(1) \in P$ for all $\xi \in \Pi_{\lambda}$. Let $\mu \in P$ be a weight, and let $V(\lambda)$ be the highest-weight module over $U_{q}(\mathfrak{g})$ of highest weight $\lambda$. A theorem of Littelmann states that the number of paths in $\xi \in \Pi_{\lambda}$, such that $\xi(1)=\mu$, is equal to the dimension of the weight space of weight $\mu$ in $V(\lambda)$ ([Littleman 95, Theorem 9,1]).

Let $\nu=\sum_{i=1}^{l} k_{i} \alpha_{i}$ be a linear combination of simple roots, with non-negative integral coefficients. Set $\lambda=$ $\sum_{i=1}^{l} k_{i} \lambda_{i}$ (where the $\lambda_{i}$ are the fundamental weights). Then the dimension of the weight space of weight $\lambda-\nu$ in $V(\lambda)$ is equal to the dimension of $U_{-\nu}^{-}$. In particular, the dimension of $U_{-\nu}^{-}$is equal to the number of paths $\xi \in \Pi_{\lambda}$ such that $\xi(1)=\lambda-\nu$.

Let $w_{0}=s_{i_{1}} \cdots s_{i_{t}}$ be a fixed reduced expression of the longest element in the Weyl group. Let $\nu, \lambda$ be as in the preceding paragraph, and let $\xi \in \Pi_{\lambda}$ be such that $\xi(1)=\lambda-\nu$. We define a sequence of integers $\eta_{\xi}=\left(n_{1}, \ldots, n_{t}\right)$ and a sequence of paths $\xi_{k}$ in the following way. First we set $\xi_{0}=\xi$. Suppose that the elements $\xi_{0}, \ldots, \xi_{k-1}$ and $n_{1}, \ldots, n_{k-1}$ are defined. Then let $n_{k}$ be maximal such that $e_{\alpha_{i_{k}}}^{n_{k}}\left(\xi_{k-1}\right) \neq 0$, and set $\xi_{k}=e_{\alpha_{i_{k}}}^{n_{k}}\left(\xi_{k-1}\right)$. Following [Littleman 98] we call $\eta_{\xi}$ the adapted string corresponding to $\xi$ (relative to the fixed reduced expression of the longest element of the Weyl group). Let $S_{\nu}$ be the set of adapted strings corresponding to all $\xi \in \Pi_{\lambda}$ such that $\xi(1)=\lambda-\nu$.

Let $\eta=\left(n_{1}, \ldots, n_{t}\right) \in S_{\nu}$ and set

$$
M_{\eta}=F_{\alpha_{i_{1}}}^{\left(n_{1}\right)} \cdots F_{\alpha_{i_{t}}}^{\left(n_{t}\right)}
$$

and

$$
b_{\eta}=\widetilde{F}_{\alpha_{i_{1}}}^{n_{1}} \ldots \widetilde{F}_{\alpha_{i_{t}}}^{n_{t}}(1) \in \mathcal{B}(\infty)
$$

Let $<_{\text {lex }}$ be the lexicographical ordering on integer sequences of length $t$ (i.e., $\left(m_{1}, \ldots, m_{t}\right)<_{\text {lex }}\left(n_{1}, \ldots, n_{t}\right)$ if there is a $k$ such that $m_{i}=n_{i}$ for $i<k$, and $\left.m_{k}<n_{k}\right)$. Then [Littleman 98, Proposition 10.4] states

$$
M_{\eta}=G\left(b_{\eta}\right)-\sum_{\substack{\eta^{\prime}>\text { lex } \\ \eta^{\prime} \in S_{\nu}}} c_{\eta, \eta^{\prime}} G\left(b_{\eta^{\prime}}\right),
$$

where $c_{\eta, \eta^{\prime}} \in \mathbb{Z}\left[q, q^{-1}\right]$.

Let $\eta=\left(n_{1}, \ldots, n_{t}\right)$ be an adapted string, corresponding to the reduced expression $w_{0}=s_{i_{1}} \cdots s_{i_{t}}$ of the longest element in $W(\Phi)$. Then we also write $f^{\eta}\left(\xi_{\lambda}\right)$ instead of $f_{\alpha_{i_{1}}}^{n_{1}} \cdots f_{\alpha_{i_{t}}}^{n_{t}}\left(\xi_{\lambda}\right)$. 


\section{CONSTRUCTING CANONICAL BASIS ELEMENTS}

Here we describe an algorithm for computing the elements of the canonical basis of a given weight $\nu$. The main idea is similar to the one used in [Leclerc and Toffin 00]: We "approximate" $G\left(b_{\eta}\right)$ with a bar-invariant element, i.e., $M_{\eta}$. Then we add multiples of the $G\left(b_{\eta^{\prime}}\right)$ that are already constructed, while making sure that the element remains bar-invariant. We are finished when the element is a $\mathbb{Z}[q]$-linear combination of PBW-monomials, where exactly one coefficient is 1 , and the rest lies in $q \mathbb{Z}[q]$.

By $<_{\text {lex }}$, we denote the lexicographical ordering on the PBW-monomials of $U^{-}$(i.e., $F_{1}^{\left(m_{1}\right)} \cdots F_{t}^{\left(m_{t}\right)}<_{\operatorname{lex}} F_{1}^{\left(n_{1}\right)} \cdots F_{t}^{\left(n_{t}\right)}$ if and only if $\left.\left(m_{1}, \ldots, m_{t}\right)<_{\text {lex }}\left(n_{1}, \ldots, n_{t}\right)\right)$.

Let $x$ be a PBW-monomial. From Section 2, we recall that $b_{x}$ denotes the element of $\mathcal{B}(\infty)$ such that $G\left(b_{x}\right)$ has principal monomial $x$. Also by $\varepsilon_{\alpha}(x)$, we denote the maximal integer $n$ such that $\widetilde{E}_{\alpha}^{n}\left(b_{x}\right) \neq 0$. Note that if $x$ is a $w_{0}$-monomial, where $w_{0}$ starts with $s_{\alpha}$, then $\varepsilon_{\alpha}(x)$ is equal to the first exponent of $x$.

Proposition 5.1. Let $w=s_{\alpha_{i_{1}}} \cdots s_{\alpha_{i_{r}}}$ be a reduced word in the Weyl group of $\Phi$. Let $w_{0}$ be any reduced expression for the longest element in the Weyl group starting with w. Let

$$
x=F_{\alpha_{i_{1}}}^{\left(n_{1}\right)} T_{\alpha_{i_{1}}}\left(F_{\alpha_{i_{2}}}\right)^{\left(n_{2}\right)} \cdots\left(T_{\alpha_{i_{1}}} \cdots T_{\alpha_{i_{r-1}}}\right)\left(F_{\alpha_{i_{r}}}\right)^{\left(n_{r}\right)}
$$

be a PBW-monomial in $U^{-}$. Then $G\left(b_{x}\right)$ is equal to $x$ plus a $q \mathbb{Z}[q]$-linear combination of $w_{0}$-monomials y such that $y>_{\text {lex }} x$.

In the proof, we use two direct sum decompositions of $U^{-}$relative to a simple root $\alpha$ :

$$
\begin{gathered}
U^{-}=U^{-} \cap T_{\alpha}\left(U^{-}\right) \oplus F_{\alpha} U^{-}, \\
U^{-}=U^{-} \cap T_{\alpha}^{-1}\left(U^{-}\right) \oplus U^{-} F_{\alpha},
\end{gathered}
$$

(cf. [Jantzen 96a, 8.25], [Lusztig 96]). We have the corresponding projection maps $\pi_{\alpha}^{+}: U^{-} \rightarrow U^{-} \cap T_{\alpha}\left(U^{-}\right)$and $\pi_{\alpha}^{-}: U^{-} \rightarrow U^{-} \cap T_{\alpha}^{-1}\left(U^{-}\right)$. These maps can be described as follows. Let $w_{0}=s_{\alpha_{i_{1}}} \cdots s_{\alpha_{i_{t}}}$ be a reduced expression for the longest element in the Weyl group, where $\alpha_{i_{1}}=\alpha$. If $(2-1)$ is a $w_{0}$-monomial, then $F_{1}=F_{\alpha}$ and $F_{\alpha} U^{-}$is the linear span of all $w_{0}$-monomials with first exponent $\geq 1$. Also $U^{-} \cap T_{\alpha}\left(U^{-}\right)$is the linear span of all $w_{0}$-monomials with first exponent equal to 0 . Now let $u \in U^{-}$and write $u$ as a linear combination of $w_{0}$-monomials. Then $u=u_{1}+u_{2}$, where $u_{1}$ consists of $w_{0}$-monomials with first exponent 0 , and $u_{2}$ is a linear combination of $w_{0^{-}}$ monomials with first exponent $\geq 1$. Hence $\pi_{\alpha}^{+}(u)=u_{1}$.

Set $v=s_{\alpha_{i_{2}}} \cdots s_{\alpha_{i_{t}}}$, and let $\beta$ be a simple root such that $v(\beta)>0$. We set $\widetilde{w}_{0}=v s_{\beta}$; then $\widetilde{w}_{0}$ is also a reduced expression for the longest element of the Weyl group. We have $v(\beta)>0$, but $s_{\alpha} v(\beta)<0$, so that $v(\beta)=\alpha$. Hence $T_{v}\left(F_{\beta}\right)=F_{\alpha}$ (cf. [Jantzen 96a, Proposition 8.20]). So if we have a $\widetilde{w}_{0}$-monomial of the form $(2-1)$, then $F_{t}=$ $F_{\alpha}$; hence $U^{-} F_{\alpha}$ is the linear span of all $\widetilde{w}_{0}$-monomials with $t^{\text {th }}$ exponent $\geq 1$. Furthermore, $U^{-} \cap T_{\alpha}^{-1}\left(U^{-}\right)$is the linear span of all $\widetilde{w}_{0}$-monomials with $t^{\text {th }}$ exponent equal to 0 . This means that we can decompose $u \in U^{-}$ according to the decomposition (5-2) by writing $u=u_{1}+$ $u_{2}$, where $u_{1}$ is a linear combination of $\widetilde{w}_{0}$-monomials with $t^{\text {th }}$ exponent 0 , and $u_{2}$ consists of $\widetilde{w}_{0}$-monomials with $t^{\text {th }}$ exponent $\geq 1$. Then $\pi_{\alpha}^{-}(u)=u_{1}$.

$B_{\alpha}^{+}=\pi_{\alpha}^{+}\left(\mathbf{B} \backslash \mathbf{B} \cap F_{\alpha} U^{-}\right)$is a basis of $U^{-} \cap T_{\alpha}\left(U^{-}\right)$, and $B_{\alpha}^{-}=\pi_{\alpha}^{-}\left(\mathbf{B} \backslash \mathbf{B} \cap U^{-} F_{\alpha}\right)$ is a basis of $U^{-} \cap T_{\alpha}^{-1}\left(U^{-}\right)$ (see [Lusztig 96]). Theorem 1.2 in [Lusztig 96] states that

$$
T_{\alpha}\left(B_{\alpha}^{-}\right)=B_{\alpha}^{+}
$$

Proof: (of Proposition 5.1). We use induction on $r$. Note that the result is trivial for $r=1$ as in that case $x=F_{\alpha_{i_{1}}}^{\left(n_{1}\right)}$ and $G\left(b_{x}\right)=x$. Set $\alpha=\alpha_{i_{1}}$ and

$$
\begin{gathered}
x^{\prime}=T_{\alpha_{i_{1}}}\left(F_{\alpha_{i_{2}}}\right)^{\left(n_{2}\right)} \cdots\left(T_{\alpha_{i_{1}}} \cdots T_{\alpha_{i_{r-1}}}\right)\left(F_{\alpha_{i_{r}}}\right)^{\left(n_{r}\right)}, \\
x^{\prime \prime}=F_{\alpha_{i_{2}}}^{\left(n_{2}\right)} T_{\alpha_{i_{2}}}\left(F_{\alpha_{i_{3}}}\right)^{\left(n_{3}\right)} \cdots\left(T_{\alpha_{i_{2}}} \cdots T_{\alpha_{i_{r-1}}}\right)\left(F_{\alpha_{i_{r}}}\right)^{\left(n_{r}\right)} .
\end{gathered}
$$

(So that $x^{\prime}=T_{\alpha}\left(x^{\prime \prime}\right)$.) We define $\widetilde{w}_{0}$ as above. Then $x^{\prime \prime}$ is a $\widetilde{w}_{0}$-monomial and by induction $G\left(b_{x^{\prime \prime}}\right)$ is equal to $x^{\prime \prime}$ plus a $q \mathbb{Z}[q]$-linear combination of $\widetilde{w}_{0}$-monomials that are lexicographically bigger than $x^{\prime \prime}$. By the description of $\pi_{\alpha}^{-}$, we see that the same holds for $\pi_{\alpha}^{-}\left(G\left(b_{x^{\prime \prime}}\right)\right)$. Now, by (5-3), $T_{\alpha}\left(\pi_{\alpha}^{-}\left(G\left(b_{x^{\prime \prime}}\right)\right)\right)=\pi_{\alpha}^{+}\left(G\left(b_{y}\right)\right)$ for some $G\left(b_{y}\right) \in \mathbf{B} \backslash \mathbf{B} \cap F_{\alpha} U^{-}$. But $T_{\alpha}\left(\pi_{\alpha}^{-}\left(G\left(b_{x^{\prime \prime}}\right)\right)\right)$ is equal to $T_{\alpha}\left(x^{\prime \prime}\right)=x^{\prime}$ plus a $q \mathbb{Z}[q]$-linear combination of $w_{0^{-}}$ monomials (lexicographically bigger than $x^{\prime}$ ), and therefore $y=x^{\prime}$. It follows that $\pi_{\alpha}^{+}\left(G\left(b_{x^{\prime}}\right)\right)$ is equal to $x^{\prime}$ plus a $q \mathbb{Z}[q]$-linear combination of $w_{0}$-monomials that are lexicographically bigger than $x^{\prime}$. From the description above of the map $\pi_{\alpha}^{+}$, we now see that $G\left(b_{x^{\prime}}\right)$ is equal to $\pi_{\alpha}^{+}\left(G\left(b_{x^{\prime}}\right)\right)$ plus a linear combination of $w_{0}$-monomials with nonzero first exponent, and these are lexicographically bigger than $x^{\prime}$. Now by [Jantzen 96a, 11.12(1)], $G\left(b_{x}\right)=F_{\alpha}^{\left(n_{1}\right)} G\left(b_{x^{\prime}}\right)+R$ where $R$ is a linear combination of elements $G\left(b_{z}\right)$, with $\varepsilon_{\alpha}(z)>n_{1}$. By [Jantzen 96a, 11.3(2), 11.12(3)], $G\left(b_{u}\right) \in F_{\alpha}^{\left(\varepsilon_{\alpha}(u)\right)} U^{-}$for all PBWmonomials $u$. In particular, all $w_{0}$-monomials occurring in $R$ have first exponent $>n_{1}$, and therefore they are bigger than $x$ in the lexicographical ordering. 
Proposition (5.1) yields the following algorithm for constructing elements of the canonical basis. From (4-1) we get:

$$
G\left(b_{\eta}\right)=M_{\eta}+\sum_{\eta^{\prime}>\operatorname{lex} \eta} c_{\eta, \eta^{\prime}} G\left(b_{\eta^{\prime}}\right) .
$$

The $M_{\eta}$ and $G\left(b_{\eta}\right)$ are all bar-invariant, and the latter form a basis of $U_{-\nu}^{-}$, hence the $c_{\eta, \eta^{\prime}}$ are bar-invariant as well.

Let $\eta \in S_{\nu}$, and suppose that we have already constructed the elements $G\left(b_{\eta^{\prime}}\right)$ for $\eta^{\prime}>_{\text {lex }} \eta$. In order to construct $G\left(b_{\eta}\right)$, we need to know the coefficients $c_{\eta, \eta^{\prime}}$ in (5-4). For $b_{1}, b_{2} \in \mathcal{B}(\infty)$, we write $b_{1}<_{\operatorname{lex}} b_{2}$ if the principal monomial of $G\left(b_{1}\right)$ is smaller with respect to $<_{\text {lex }}$ than the principal monomial of $G\left(b_{2}\right)$. Order the elements occurring in the sum on the righthand side of $(5-4)$ as $b_{\eta_{1}}<_{\text {lex }} b_{\eta_{2}}<_{\text {lex }} \cdots<_{\text {lex }} b_{\eta_{r}}$. We define a sequence of elements $G_{k} \in U^{-}$. First set $G_{0}=M_{\eta}$. Suppose that $G_{0}, \ldots, G_{k-1}$ are defined. Let $\zeta_{k}$ be the coefficient of the principal monomial of $G\left(b_{\eta_{k}}\right)$ in $G_{k-1}$, and let $\zeta_{k}^{\prime}$ be the unique bar-invariant element of $\mathbb{Z}\left[q, q^{-1}\right]$ such that $\zeta_{k}+\zeta_{k}^{\prime} \in q \mathbb{Z}[q]$. Set $G_{k}=G_{k-1}+\zeta_{k}^{\prime} G\left(b_{\eta_{k}}\right)$. By induction on $k$, and Proposition 5.1, $c_{\eta, \eta_{k}}=\zeta_{k}^{\prime}$. Hence $G_{r}=G\left(b_{\eta}\right)$.

Example 5.2. We consider the root system of type $B_{2}$, with simple roots $\alpha$ and $\beta$, where $\alpha$ is long. We use the reduced expression $s_{\alpha} s_{\beta} s_{\alpha} s_{\beta}$ of the longest element in the Weyl group. The generators of the corresponding PBW-type basis of $U^{-}$are $F_{\alpha}, F_{\alpha+\beta}, F_{\alpha+2 \beta}, F_{\beta}$. Let $\nu=3 \alpha+2 \beta$; we compute the elements of the canonical basis of weight $\nu$.

The set $S_{\nu}$ consists of the adapted strings $\eta_{1}=$ $(3,2,0,0), \quad \eta_{2}=(2,2,1,0), \quad \eta_{3}=(2,1,1,1), \quad \eta_{4}=$ $(1,2,2,0)$ (in lexicographical order). We know that $M_{\eta_{1}}=F_{\alpha}^{(3)} F_{\beta}^{(2)}=G\left(b_{\eta_{1}}\right)$. Now we consider $\eta_{2}$. Using the algorithms to compute products of PBW-monomials in $U^{-}$([Graaf 01a]), which are implemented in [Graaf $01 \mathrm{~b}]$, we get

$$
\begin{aligned}
M_{\eta_{2}} & =F_{\alpha}^{(2)} F_{\beta}^{(2)} F_{\alpha} \\
& =F_{\alpha}^{(2)} F_{\alpha+2 \beta}+q F_{\alpha}^{(2)} F_{\alpha+\beta} F_{\beta}+\left(1+q^{4}+q^{8}\right) F_{\alpha}^{(3)} F_{\beta}^{(2)} .
\end{aligned}
$$

Here the coefficient of $F_{\alpha}^{(3)} F_{\beta}^{(2)}$ is not contained in $q \mathbb{Z}[q]$. We repair this situation, and we get

$$
\begin{aligned}
G\left(b_{\eta_{2}}\right) & =M_{\eta_{2}}-G\left(b_{\eta_{1}}\right) \\
& =F_{\alpha}^{(2)} F_{\alpha+2 \beta}+q F_{\alpha}^{(2)} F_{\alpha+\beta} F_{\beta}+\left(q^{4}+q^{8}\right) F_{\alpha}^{(3)} F_{\beta}^{(2)} .
\end{aligned}
$$

Since

$M_{\eta_{3}}=F_{\alpha}^{(2)} F_{\alpha+\beta} F_{\beta}+\left(q^{-3}+q^{-1}+q+q^{3}+q^{5}+q^{7}\right) F_{\alpha}^{(3)} F_{\beta}^{(2)}$, we have

$$
\begin{aligned}
G\left(b_{\eta_{3}}\right) & =M_{\eta_{3}}-\left(q^{-3}+q^{-1}+q+q^{3}\right) G\left(b_{\eta_{1}}\right) \\
& =F_{\alpha}^{(2)} F_{\alpha+\beta} F_{\beta}+\left(q^{5}+q^{7}\right) F_{\alpha}^{(3)} F_{\beta}^{(2)} .
\end{aligned}
$$

Finally,

$$
\begin{aligned}
M_{\eta_{4}}= & F_{\alpha} F_{\alpha+\beta}^{(2)}+\left(1+q^{4}\right) F_{\alpha}^{(2)} F_{\alpha+2 \beta} \\
& +\left(q+q^{5}\right) F_{\alpha}^{(2)} F_{\alpha+\beta} F_{\beta}+\left(q^{4}+q^{8}+q^{12}\right) F_{\alpha}^{(3)} F_{\beta}^{(2)} .
\end{aligned}
$$

Here the coefficient of $F_{\alpha}^{(2)} F_{\alpha+2 \beta}$ does not lie in $q \mathbb{Z}[q]$. So we have to subtract the principal monomial, $G\left(b_{\eta_{2}}\right)$, from the element of the canonical basis. We get

$$
\begin{aligned}
G\left(b_{\eta_{4}}\right)= & M_{\eta_{4}}-G\left(b_{\eta_{2}}\right) \\
= & F_{\alpha} F_{\alpha+\beta}^{(2)}+q^{4} F_{\alpha}^{(2)} F_{\alpha+2 \beta}+q^{5} F_{\alpha}^{(2)} F_{\alpha+\beta} F_{\beta} \\
& +q^{12} F_{\alpha}^{(3)} F_{\beta}^{(2)} .
\end{aligned}
$$

As a first application of the algorithm for constructing elements of the canonical basis, we give an algorithm for constructing highest-weight modules. Let $\lambda$ be a dominant weight. Let $v_{\lambda}$ be a highest-weight vector of the highest weight module $V(\lambda)$. Then according to [Jantzen 96a, Theorem $11.10(\mathrm{~d})]$, the set $\left\{G(b) \cdot v_{\lambda} \mid b \in\right.$ $\mathcal{B}(\infty)\} \backslash\{0\}$ is a basis of $V(\lambda)$. Using the path method, it is straightforward to decide which $b \in \mathcal{B}(\infty)$ satisfy $G(b) \cdot v_{\lambda}=0$. Let $b=b_{\eta}$ for some adapted string $\eta$. Then $G(b) \cdot v_{\lambda}=0$ if and only if $f^{\eta} \xi_{\lambda}=0$ (this follows from Lemma 6.1, along with [Jantzen 96a, Theorem $11.10(\mathrm{~d})])$.

Furthermore, we only have to check that $b \in \mathcal{B}(\infty)$ with weight $\nu$ such that the multiplicity of $\lambda-\nu$ in $V(\lambda)$ is nonzero. By a standard algorithm, we can calculate the set of all those $\nu$ (using the path method, for example). Now the nonzero $G(b) \cdot v_{\lambda}$ form a basis of the highestweight module, and we use the $G(b)$ where $G(b) \cdot v_{\lambda}=0$ to rewrite all other vectors as linear combinations of basis elements. This algorithm is rather inefficient because the dimension of $U_{-\nu}^{-}$grows quickly as the level of $\nu$ increases. A more efficient algorithm for constructing highest-weight modules is indicated in [Graaf 01a]. However, using the algorithm described above, it is possible to investigate single weight spaces of a highest-weight module, without first constructing the module.

Example 5.3. We use the same notation as in Example 5.2. Let $\lambda=\lambda_{1}$ be the first fundamental weight. Then $V(\lambda)$ has a weight space of weight $-\lambda_{1}=\lambda-2 \alpha-2 \beta$. 
The elements of the canonical basis of weight $2 \alpha+2 \beta$ are

$$
\begin{aligned}
& G\left(b_{1}\right)=F_{\alpha}^{(2)} F_{\beta}^{(2)} ; \\
& G\left(b_{2}\right)=F_{\alpha} F_{\alpha+\beta} F_{\beta}+\left(q^{3}+q^{5}\right) F_{\alpha}^{(2)} F_{\beta}^{(2)} ; \\
& G\left(b_{3}\right)=F_{\alpha} F_{\alpha+2 \beta}+q F_{\alpha} F_{\alpha+\beta} F_{\beta}+\left(q^{2}+q^{6}\right) F_{\alpha}^{(2)} F_{\beta}^{(2)} ; \\
& G\left(b_{4}\right)=F_{\alpha+\beta}^{(2)}+q^{2} F_{\alpha} F_{\alpha+2 \beta}+q^{3} F_{\alpha} F_{\alpha+\beta} F_{\beta}+q^{8} F_{\alpha}^{(2)} F_{\beta}^{(2)} .
\end{aligned}
$$

They correspond to the strings $\eta_{1}=(2,2,0,0), \eta_{2}=$ $(1,1,1,1), \eta_{3}=(1,2,1,0)$ and $\eta_{4}=(0,2,2,0)$, respectively. Now only $f^{\eta_{3}} \xi_{\lambda} \neq 0$. So $G\left(b_{i}\right) \cdot v_{\lambda}=0$ for $i=1,2,4$. Let $x_{i}$ denote the principal monomial of $G\left(b_{i}\right)$. We see that $x_{i} \cdot v_{\lambda}=0$ for $i=1,2$, and $x_{4} \cdot v_{\lambda}=-q^{2} x_{3} \cdot v_{\lambda}$.

We end this section with a sketch of a proof of Case 3 of the formulas for the exponents $m_{i}^{\prime}$ in Section 3 . We consider the case where the root system is of type $B_{2}$. We let $\alpha, \beta$ be the simple roots, where $\beta$ is long. First suppose that we use the reduced expression $s_{\alpha} s_{\beta} s_{\alpha} s_{\beta}$. Then by [Littleman 98, Corollary 2], the set $C_{1}^{s, r}$ of adapted strings of weight $s \alpha+r \beta$ consists of all $\eta_{l, m}=$ $(s-m, r-l, m, l)$, such that $0 \leq m \leq s, 0 \leq l \leq r$ and $2(r-l) \geq m \geq 2 l$. Here we have $\eta_{l, m}>_{\text {lex }} \eta_{l^{\prime}, m^{\prime}}$ if $m<m^{\prime}$ or $m=m^{\prime}$ and $l<l^{\prime}$.

Now

$$
\begin{aligned}
& F_{\alpha}^{(s-m)} F_{\beta}^{(r-l)} F_{\alpha}^{(m)} F_{\beta}^{(l)}= \\
& \quad \sum_{\substack{i, j \geq 0 \\
i+j \leq r-l \\
2 i+j \leq m}} q^{(m-2 i-j)(2 r-2 l-2 i-j)+2(r-l-i-j) i} \\
& {\left[\begin{array}{c}
s-2 i-j \\
s-m
\end{array}\right]_{\alpha}\left[\begin{array}{c}
r-i-j \\
l
\end{array}\right]_{\beta} F_{\alpha}^{(s-2 i-j)} F_{2 \alpha+\beta}^{(i)} F_{\alpha+\beta}^{(j)} F_{\beta}^{(r-i-j)} .}
\end{aligned}
$$

By studying the coefficients in this expression, and following the algorithm for computing elements of the canonical basis, it can be shown that the principal monomial of $G\left(b_{\eta_{l, m}}\right)$ is

$$
\begin{array}{ccc}
F_{\alpha}^{(s-m)} F_{2 \alpha+\beta}^{(l)} F_{\alpha+\beta}^{(m-2 l)} F_{\beta}^{(r-m+l)} & \text { if } & m \leq r ; \\
F_{\alpha}^{(s-m)} F_{2 \alpha+\beta}^{(m+l-r)} F_{\alpha+\beta}^{(2 r-2 l-m)} F_{\beta}^{(l)} & \text { if } & m \geq r .
\end{array}
$$

Now suppose that we use the reduced expression $s_{\beta} s_{\alpha} s_{\beta} s_{\alpha}$. The set $C_{2}^{s, r}$ of adapted strings of weight $s \alpha+r \beta$ consists of all $\zeta_{l, m}=(r-m, s-l, m, l)$ such that $0 \leq l \leq s, 0 \leq m \leq r, s-l \geq m \geq l$ (see [Littleman 98, Corollary 2]). Thus, $\zeta_{l, m}>_{\text {lex }} \zeta_{l^{\prime}, m^{\prime}}$ if $m<m^{\prime}$ or $m=m^{\prime}$ and $l<l^{\prime}$. In this case the principal monomial of $G\left(b_{\zeta_{l, m}}\right)$ is

$$
\begin{array}{cc}
F_{\beta}^{(r-m)} F_{\alpha+\beta}^{(2 m-s+l)} F_{2 \alpha+\beta}^{(s-l+m)} F_{\alpha}^{(l)} & \text { if } \quad s \leq 2 m ; \\
F_{\beta}^{(r-m)} F_{\alpha+\beta}^{(l)} F_{2 \alpha+\beta}^{(m-l)} F_{\alpha}^{(s+l-2 m)} & \text { if } \quad s \geq 2 m .
\end{array}
$$

Suppose that the braid relation consists of replacing $s_{\alpha} s_{\beta} s_{\alpha} s_{\beta}$ by $s_{\beta} s_{\alpha} s_{\beta} s_{\alpha}$. We start with a PBW-monomial $x=F_{\alpha}^{(a)} F_{2 \alpha+\beta}^{(b)} F_{\alpha+\beta}^{(c)} F_{\beta}^{(d)}$. We form the adapted string $\eta$ such that $G\left(b_{\eta}\right)$ has principal monomial $x$. By the description of the principal monomials above, $\eta=(a, c+$ $\max (b, d), 2 b+c, \min (b, d))$. Now we use the bijection $\phi: C_{1}^{s, r} \rightarrow C_{2}^{s, r}$, such that $f^{\theta}=f^{\phi(\theta)}$ for all $\theta \in C_{1}^{s, r}$. According to [Littleman 98, Proposition 2.4], $\phi(\eta)=$ $\left(n_{1}, n_{2}, n_{3}, n_{4}\right)$, where $n_{1}=\max (b, \max (b, d)+c-a)$, $n_{2}=\max (a, c)+2 b, n_{3}=\min (c+d, a+\min (b, d))$, $n_{4}=\min (a, c)$. Now $\phi(\eta)$ corresponds to the PBWmonomial $F_{\beta}^{\left(n_{1}\right)} F_{\alpha+\beta}^{\left(2 n_{3}-n_{2}\right)} F_{2 \alpha+\beta}^{\left(n_{2}-n_{3}\right)} F_{\alpha}^{\left(n_{4}\right)}$ if $n_{2}+n_{4} \leq 2 n_{3}$, and to $F_{\beta}^{\left(n_{1}\right)} F_{\alpha+\beta}^{\left(n_{4}\right)} F_{2 \alpha+\beta}^{\left(n_{3}-n_{4}\right)} F_{\alpha}^{\left(n_{2}+2 n_{4}-2 n_{3}\right)}$ if $n_{2}+n_{4} \geq$ $2 n_{3}$. This implies the formulas in Case 3(a); Case 3(b) is similar. The formula in Case 2 can also be proved this way.

\section{CANONICAL BASES OF MODULES}

For a dominant weight $\lambda$, let $V(\lambda)$ be the finitedimensional highest-weight module over $U_{q}(\mathfrak{g})$ with highest weight $\lambda$. Let $v_{\lambda} \in V(\lambda)$ be a fixed highest weight vector. Set $\mathbf{B}(\lambda)=\left\{G(b) \cdot v_{\lambda} \mid G(b) \in \mathbf{B}\right\} \backslash\{0\}$. Then $\mathbf{B}(\lambda)$ is a basis of $V(\lambda)$ (cf. [Jantzen 96a, Theorem 11.10]), called the canonical basis of $V(\lambda)$. We can compute $\mathbf{B}(\lambda)$ by computing elements of $\mathbf{B}$. However, this method is rather inefficient, since for many $G(b) \in \mathbf{B}, G(b) \cdot v_{\lambda}=0$. In this section we describe an algorithm for computing $\mathbf{B}(\lambda)$ without first computing elements of $\mathbf{B}$.

Let $\varphi_{\lambda}: U^{-} \rightarrow V(\lambda)$ be the map defined by $\varphi_{\lambda}(u)=$ $u \cdot v_{\lambda}$. Set $\mathcal{L}(\lambda)=\varphi_{\lambda}(\mathcal{L}(\infty))$; then $\mathcal{L}(\lambda)$ is a $\mathbb{Z}[q]$-lattice in $V(\lambda)$ spanned by all nonzero $G(b) \cdot v_{\lambda}$ for $G(b) \in \mathbf{B}$.

By $\varphi_{\lambda}$, we also denote the induced map $\varphi_{\lambda}$ : $\mathcal{L}(\infty) / q \mathcal{L}(\infty) \rightarrow \mathcal{L}(\lambda) / q \mathcal{L}(\lambda)$, and we set $\mathcal{B}(\lambda)=$ $\varphi_{\lambda}(\mathcal{B}(\infty)) \backslash\{0\}$. Then $\mathcal{B}(\lambda)$ consists of all $x \cdot v_{\lambda} \bmod$ $q \mathcal{L}(\lambda)$, where $x$ runs through all PBW-monomials such that $G\left(b_{x}\right) \cdot v_{\lambda} \neq 0$. Therefore, $|\mathcal{B}(\lambda)|=\operatorname{dim} V(\lambda)$.

For $\alpha \in \Delta$, we use the Kashiwara operator $\widetilde{F}_{\alpha}$ : $V(\lambda) \rightarrow V(\lambda)$ as defined in [Jantzen 96a, 9.2]. For $u \in \mathcal{L}(\infty)$, we have $\widetilde{F}_{\alpha}\left(u \cdot v_{\lambda}\right)=\widetilde{F}_{\alpha}(u) \cdot v_{\lambda} \bmod q \mathcal{L}(\lambda)$ (where the second $\widetilde{F}_{\alpha}$ is the Kashiwara operator on $U^{-}$), cf. [Jantzen 96a, Proposition 10.9]. Therefore, $\widetilde{F}_{\alpha}$ is also a map from $\mathcal{B}(\lambda)$ into $\mathcal{B}(\lambda) \cup\{0\}$.

Let $\eta=\left(n_{1}, \ldots, n_{t}\right)$ be an adapted string, relative to the reduced expression $w_{0}=s_{\alpha_{i_{1}}} \cdots s_{\alpha_{i_{t}}}$. Then we write $\widetilde{F}^{\eta}$ for $\widetilde{F}_{\alpha_{i_{1}}}^{n_{1}} \ldots \widetilde{F}_{\alpha_{i_{t}}}^{n_{t}}$ (where the $\widetilde{F}_{\alpha_{k}}$ are the Kashiwara operators on $U^{-}$or the Kashiwara operators on $\left.V(\lambda)\right)$. From Section 4 , we recall that $\xi_{\lambda}$ denotes the path joining 0 with $\lambda$ by a straight line. 
Lemma 6.1. Let $\eta$ be an adapted string, and set $b=$ $\widetilde{F}^{\eta}(1) \in \mathcal{B}(\infty)$. Then $\varphi_{\lambda}(b)=0$ if and only if $f^{\eta}\left(\xi_{\lambda}\right)=0$.

Proof: Set $b_{\lambda}=\varphi_{\lambda}(1) \in \mathcal{B}(\lambda)$. By [Kashiwara 96, Theorem 4.1], $f^{\eta} \xi_{\lambda}=0$ if and only if $\widetilde{F}^{\eta} b_{\lambda}=0$. By [Jantzen 96a, Proposition 10.9], this is equivalent to $\varphi_{\lambda}\left(\widetilde{F}^{\eta}(1)\right)=0$.

For an adapted string $\eta$, we denote by $x_{\eta}$ the PBWmonomial with the property $\widetilde{F}^{\eta}(1)=x_{\eta} \bmod q \mathcal{L}(\infty)$. Note that we can compute $x_{\eta}$ by using the algorithm for computing the action of $\widetilde{F}_{\alpha}$, described in Section 3 . So Lemma 6.1 gives a straightforward algorithm for computing the elements of $\mathcal{B}(\lambda)$ of a given weight $\lambda-\nu$. We loop over all $\eta \in S_{\nu}$ and for every $\eta$ such that $f^{\eta} \xi_{\lambda} \neq 0$, we compute $x_{\eta} \cdot v_{\lambda} \bmod q \mathcal{L}(\lambda)$.

By -, we denote the involution of $V(\lambda)$ defined by $\overline{u \cdot v_{\lambda}}=\bar{u} \cdot v_{\lambda}$, for $u \in U^{-}$(this is well defined by [Jantzen 96a, Proposition 11.9 (b)]).

Lemma 6.2. Let $b \in \mathcal{B}(\lambda)$. Then there is a unique element $v(b) \in \mathcal{L}(\lambda)$ such that $v(b)=b \bmod q \mathcal{L}(\lambda)$ and $\overline{v(b)}=v(b)$. Let $b^{\prime} \in \mathcal{B}(\infty)$ be such that $\varphi_{\lambda}\left(b^{\prime}\right)=b$; then $v(b)=\varphi_{\lambda}\left(G\left(b^{\prime}\right)\right)$.

Proof: It is clear that $\varphi_{\lambda}\left(G\left(b^{\prime}\right)\right)$ has the listed properties. Suppose that the element $v \in \mathcal{L}(\lambda)$ also has these properties. Then we write $v$ as a linear combination of elements $\varphi_{\lambda}\left(G\left(b^{\prime \prime}\right)\right)$. Because $v$ is bar-invariant, the coefficients in this expression must be bar-invariant as well. Because the $\varphi_{\lambda}\left(G\left(b^{\prime \prime}\right)\right)$ form a basis of $\mathcal{L}(\lambda)$ over $\mathbb{Z}[q]$, the coefficients must lie in $\mathbb{Z}[q]$. This means that the coefficients are elements of $\mathbb{Z}$. Since $v=b \bmod q \mathcal{L}(\lambda)$, the only $\varphi_{\lambda}\left(G\left(b^{\prime \prime}\right)\right)$ that has a nonzero coefficient is $\varphi_{\lambda}\left(G\left(b^{\prime}\right)\right)$.

Let $\nu$ be a weight such that $\lambda-\nu$ is a weight of $V(\lambda)$. Let $\xi_{1}, \ldots, \xi_{r}$ be the paths in $\Pi_{\lambda}$ ending in $\lambda-\nu$. Let $\widetilde{S}_{\nu}=\left\{\eta_{1}, \ldots, \eta_{r}\right\}$ be the corresponding adapted strings (relative to some fixed reduced expression for the longest element in the Weyl group). Note that by [Littleman 98, Lemma 1.3], $\widetilde{S}_{\nu}$ is the set of all $\eta \in S_{\nu}$ (defined as in Section 4) such that $f^{\eta} \xi_{\lambda} \neq 0$. $\widetilde{S}_{\nu}$

By taking images under $\varphi_{\lambda}$, we get by (5-4), for $\eta \in$

$$
v\left(b_{\eta}\right)=M_{\eta} \cdot v_{\lambda}+\sum_{\eta^{\prime}>\operatorname{lex} \eta} c_{\eta, \eta^{\prime}} v\left(b_{\eta^{\prime}}\right) .
$$

For $1 \leq i \leq r$, set $u_{i}=x_{\eta_{i}} \cdot v_{\lambda}$, and write $u_{i}<_{\operatorname{lex}} u_{j}$ if $x_{\eta_{i}}<$ lex $x_{\eta_{j}}$. Then Proposition 5.1 implies that

$$
v\left(b_{\eta_{i}}\right)=u_{i}+\sum_{u_{i_{k}}<\operatorname{lex} u_{i}} \zeta_{i, i_{k}} u_{i_{k}}
$$

where all $\zeta_{i, i_{k}} \in q \mathbb{Z}[q]$. We call $u_{i}$ the principal vector of $v\left(b_{\eta_{i}}\right)$.

By Lemma $6.2, v\left(b_{\eta_{i}}\right)$ is the unique bar-invariant element of $\mathcal{L}(\lambda)$ of the form of the righthand side of $(6-2)$. (Note that all $u_{i} \in \mathcal{L}(\lambda)$, so that any vector of the form $(6-2)$ belongs to $\mathcal{L}(\lambda)$.) Hence by $(6-1)$, and $(6-2)$, we have an algorithm for computing the $v\left(b_{\eta_{i}}\right)$ that is highly analogous to the algorithm for computing elements of the canonical basis of $U^{-}$. The $u_{i}$ play the role of the PBWmonomials, and we use principal vectors instead of principal monomials. Furthermore, the role of $S_{\nu}$ is taken by $\widetilde{S}_{\nu}$, and $M_{\eta}$ is replaced by $M_{\eta} \cdot v_{\lambda}$. The details of the algorithm are exactly the same; we leave them to the reader.

Note that here we do not need to use the algorithm for multiplying PBW-monomials in $U^{-}$(this is in contrast to the algorithm given in Section 5). We only need to be able to compute the action of any given PBW-monomial on elements of the module $V(\lambda)$.

Remark 6.3. By the results in [Frenkel et al. 98], the algorithm for computing canonical bases of modules can, in principle, be used to compute Kazhdan-Lusztig polynomials in the $A_{n}$-case. However, since there are specialized methods available for that, it seems unlikely that such an algorithm will beat the existing ones (see [du Cloux 96]).

\section{TIGHT MONOMIALS OF SMALL WEIGHT}

Following [Lusztig 93b], we call a monomial $m=$ $F_{\alpha_{i_{1}}}^{\left(n_{1}\right)} \cdots F_{\alpha_{i_{r}}}^{\left(n_{r}\right)}$ tight if $m \in \mathbf{B}$. The canonical bases of the quantized enveloping algebras of types $A_{1}, A_{2}$ consist entirely of tight monomials. But this is not the case for most other types. In this section, we study (experimentally) the number of elements of $\mathbf{B}$ of small weight that are tight monomials for a few examples of root systems. (We note that different monomials can define the same element of $U^{-}$, so that the number of tight monomials is usually higher than the number of elements of $\mathbf{B}$ that are tight monomials.)

We use a rather crude algorithm for computing all tight monomials of a given weight $\nu$. First, we write down all monomials in the generators $F_{\alpha_{i}}$ of weight $\nu$. Then, we calculate the set $\mathbf{B}_{\nu}$ of elements of the canonical basis of weight $\nu$. Finally, we check which monomials are contained in $\mathbf{B}_{\nu}$, by writing each monomial on the PBWtype basis used to represent the elements of $\mathbf{B}_{\nu}$.

We consider the root systems of type $D_{4}, E_{6}, F_{4}$, and $G_{2}$. In each case, for a few small weights, we compute the number of elements of $\mathbf{B}$ that are tight monomials. 


\begin{tabular}{|c|c|r|r|r|}
\hline type & weight $\nu$ & $\operatorname{dim} U_{\nu}^{-}$ & time (s) & tight \\
\hline$D_{4}$ & $(2,2,2,2)$ & 37 & 2.6 & 37 \\
$D_{4}$ & $(1,2,3,4)$ & 27 & 5.5 & 23 \\
$D_{4}$ & $(3,2,3,3)$ & 37 & 6.4 & 27 \\
$D_{4}$ & $(3,3,3,3)$ & 128 & 78.6 & 120 \\
\hline$E_{6}$ & $(1,1,2,2,1,1)$ & 110 & 8.1 & 110 \\
$E_{6}$ & $(1,1,2,3,1,1)$ & 146 & 21.4 & 116 \\
$E_{6}$ & $(1,1,2,3,2,1)$ & 354 & 112.0 & 318 \\
\hline$F_{4}$ & $(1,3,2,1)$ & 28 & 1.5 & 18 \\
$F_{4}$ & $(2,2,2,2)$ & 50 & 3.9 & 50 \\
$F_{4}$ & $(1,2,3,4)$ & 84 & 32.5 & 64 \\
$F_{4}$ & $(4,3,2,1)$ & 56 & 8.7 & 48 \\
\hline$G_{2}$ & $(3,3)$ & 8 & 0.7 & 6 \\
$G_{2}$ & $(4,4)$ & 13 & 12.4 & 8 \\
$G_{2}$ & $(5,5)$ & 20 & 1017.2 & 10 \\
\hline
\end{tabular}

TABLE 1. The number of tight monomials of small weight. The third column lists the dimension of $U_{\nu}^{-}$, and the fourth column the time taken (in seconds) to calculate $\mathbf{B}_{\nu}$. The last column contains the number of elements of $\mathbf{B}_{\nu}$ that are tight monomials.

The computations were done using the GAP4 package QuaGroup, on a Linux system with a $600 \mathrm{MHz}$ Pentium III processor and $32 \mathrm{MB}$ of working memory for GAP. The results are listed in Table 1 , where we represent a weight by giving its coefficients when written as a linear combination of simple roots. We have used the same ordering of simple roots as in [Bourbaki 68].

We see that the time needed to compute $\mathbf{B}_{\nu}$ can increase rapidly when the height of $\nu$ increases. This is seen most dramatically in the case of $G_{2}$. The most timeconsuming part of the algorithm is the "straightening" algorithm, that writes the monomials $M_{\eta}$ as linear combinations of PBW-monomials. The average cost of this algorithm is less for the higher rank cases.

In all cases, except $G_{2}$, by far the most elements of $\mathbf{B}_{\nu}$ are tight monomials. The question is whether this remains true for weights of higher level. In the case of $G_{2}$, the percentage of tight monomials drops rather sharply as the level of $\nu$ increases.

\section{REFERENCES}

[Berenstein and Zelevinsky 01] A. Berenstein and A. Zelevinsky. "Tensor product multiplicities, canonical bases and totally positive varieties." Invent. Math. 143:1 (2001), $77-128$.

[Bourbaki 68] N. Bourbaki. Groupes et Algèbres de Lie, Chapitres 4, 5 et 6. Hermann, Paris, 1968.
[Carter 97] R. W. Carter. "Canonical bases, reduced words, and Lusztig's piecewise-linear function." in Algebraic groups and Lie groups, pp. 61-79, Cambridge Univ. Press, Cambridge, 1997.

[du Cloux 96] F. du Cloux. "The state of the art in the computation of Kazhdan-Lusztig polynomials." Appl. Algebra Engrg. Comm. Comput. 7:3 (1996), 211-219.

[Frenkel et al. 98] I. B. Frenkel, M. G. Khovanov, and A. A. Kirillov, Jr. "Kazhdan-Lusztig polynomials and canonical basis." Transform. Groups 3:4 (1998), 321-336.

[GAP 00] The GAP Group. GAP - Groups, Algorithms, and Programming, Version 4.2, Aachen/St Andrews, 2000. (http://www-gap.dcs.st-and.ac.uk/ gap).

[Graaf 01a] W. A. de Graaf. "Computing with quantized enveloping algebras: PBW-type bases, highest-weight modules, $R$-matrices." J. Symbolic Comput. 32:5 (2001), $475-490$

[Graaf 01b] W. A. de Graaf. QuaGroup a GAP share package, 2001. (www.math.uu.nl/people/graaf/quagroup.html).

[Jantzen 96a] J. C. Jantzen. Lectures on Quantum Groups, volume 6 of Graduate Studies in Mathematics. Amer. Math. Soc., Providence, RI, 1996.

[Kashiwara 96] M. Kashiwara. "Similarity of crystal bases." in Lie algebras and their representations (Seoul, 1995), pp. 177-186, Amer. Math. Soc., Providence, RI, 1996.

[Leclerc and Toffin 00] B. Leclerc and P. Toffin. "A simple algorithm for computing the global crystal basis of an irreducible $U_{q}\left(\mathrm{sl}_{n}\right)$-module." Internat. J. Algebra Comput. 10:2 (2000), 191-208.

[Littleman 95] P. Littelmann. "Paths and root operators in representation theory." Ann. of Math. (2), 142:3 (1995) $499-525$.

[Littleman 98] P. Littelmann. "Cones, crystals, and patterns." Transform. Groups 3:2 (1998), 145-179.

[Lusztig 90] G. Lusztig. "Canonical bases arising from quantized enveloping algebras." J. Amer. Math. Soc. 3:2 (1990), 447-498.

[Lusztig 92] G. Lusztig. "Introduction to quantized enveloping algebras." in New developments in Lie theory and their applications (Córdoba, 1989), pp. 49-65. Birkhäuser Boston, Boston, MA, 1992.

[Lusztig 93a] G. Lusztig. Introduction to quantum groups. Birkhäuser Boston Inc., Boston, MA, 1993.

[Lusztig 93b] G. Lusztig. "Tight monomials in quantized enveloping algebras." in Quantum deformations of algebras and their representations (Ramat-Gan, 1991/1992; Rehovot, 1991/1992), pp. 117-132, Bar-Ilan Univ., Ramat Gan, 1993. 
[Lusztig 96] G. Lusztig. "Braid group action and canonical bases." Adv. Math. 122:2 (1996), 237-261.

[Marsh 96] R. J. Marsh. "Algorithms to obtain the canonical basis in some fundamental modules of quantum groups." J. Algebra 186:3 (1996), 831-860.
[Xi 99a] N. Xi. "Canonical basis for type $A_{3}$." Comm. Algebra 27:11 (1999), 5703-5710.

[Xi 99b] N. Xi. "Canonical basis for type $B_{2}$." J. Algebra 214:1 (1999), 8-21.

Willem A. de Graaf, Mathematical Institute, University of Utrecht, P.O. Box 80010, 3508 TA Utrecht, The Netherlands (degraaf@math.uu.nl)

Received October 2, 2001; accepted in revised form November 13, 2001. 